ÉGYPTE monde arabe

\section{Égypte/Monde arabe}

$7 \mid 2010$

Fabrique des élections

\title{
Splendeurs et misères du clientélisme
}

\section{Tewfik Aclimandos}

\section{CpenEdition}

\section{Journals}

Édition électronique

URL : https://journals.openedition.org/ema/3023

DOI : 10.4000/ema.3023

ISSN : 2090-7273

\section{Éditeur}

CEDEJ - Centre d'études et de documentation économiques juridiques et sociales

\section{Édition imprimée}

Date de publication : 31 décembre 2010

Pagination : 197-219

ISBN : 978-2-905838-45-2

ISSN : 1110-5097

\section{Référence électronique}

Tewfik Aclimandos, "Splendeurs et misères du clientélisme », Égypte/Monde arabe [En ligne], 7 | 2010, mis en ligne le 01 juin 2012, consulté le 07 juillet 2022. URL : http://journals.openedition.org/ema/ 3023 ; DOl : https://doi.org/10.4000/ema.3023 


\section{Tewfik Aclimandos}

\section{SPLENDEURS ET MISÈRES DU CLIENTÉLISME}

eci est un travail en cours, inachevé, frustrant. Inachevé et frustrant
parce que je n'ai pas, au terme de quelques mois de travail, réussi à " prendre connaissance » de tous les matériaux disponibles. La presse, si on effectue des vérifications, si on la compare avec les monographies de quartier ou de village, si on la lit avec un certain recul dans le temps, à froid, après que la poussière de la bataille soit retombée, fourmille d'enseignements. Les enquêtes de terrain, si elles ont le tort de vouloir en général construire des schémas généraux à partir d'une étude de cas dont la validité n'est que partielle, sont souvent passionnantes et instructives.

Je ne suis ni un spécialiste de sociologie électorale, ni du vote, ni des études parlementaires. Ma légitimité est donc limitée, mais j'ai quelques points forts:

- Il m'est arrivé d'accompagner pendant plusieurs mois, d'être le confident d'une "machine à gagner des élections », combinant charisme et réseaux clientélistes, le doyen Hâshim Fuâd (I'ancien « patron » du Centre Hospitalier Universitaire du Caire et PDG du très huppé Guézireh Sporting Club). Certes, les campagnes que j'ai préparées avec lui n'étaient pas des législatives, mais des élections à des syndicats professionnels (il décida de ne pas se présenter) ou à la présidence de clubs sportifs (il les remporta à deux reprises). Je suis très conscient de la différence de nature entre ces élections (j'en cite deux : absence des plus défavorisés, absence ou degrés différents de violence) mais il reste que l'on peut dire que j'ai mis la main à la pâte et que je cerne quelques contraintes de l'exercice ;

- Le deuxième point fort est un certain recul historique, j'ai fait deux terrains en 1990 et 1991 (élections en Haute Égypte, Hawatka et Assiout en 1990, plus entretiens semi-directifs nombreux avec un échantillon aléatoire, mais assez diversifié, suite aux victoires du FIS algérien). J'ai eu, au courant de mes pérégrinations et mondanités, des conversations très prolongées, pendant les années quatre-vingt dix, avec trois députés en vue... ;

- Par contre, si on excepte un travail sur les Frères musulmans, je n'ai pas fait de véritable terrain récemment. Mais j'ai lu la presse avec attention et j'ai eu, un peu par hasard, de longues conversations avec deux députés sortant, et, d'un autre côté, avec un candidat (qui a été battu lors des élections législatives de 2005). 
En d'autres termes, je ne suis pas un spécialiste, mais j'ai eu un accès privilégié au savoir conventionnel, exact ou non, des professionnels. Et je n'ai pas retrouvé les questions que je me suis posées dans les travaux en cours. Elles sont peut-être mauvaises, mais je préfère les poser.

Mes présupposés sont naïfs et je préfère les assumer. Pour moi, une intelligence correcte des mécanismes sociaux ne peut se passer d'une vision correcte de ou des ensembles où ils s'insèrent, de la « totalité » dans ce qu'elle a de commun avec d'autres «totalités » et aussi par ce qu'elle a de spécifique. II ne s'agit pas de dire que le tout détermine la partie. Mais d'affirmer que la partie ne se comprend que dans le cadre du tout. L'autre présupposé choquera davantage : les processus électoraux étant (en Égypte) ce qu'ils sont, il est clair qu'il ne suffira pas d'organiser des élections sans ingérence étatique pour instaurer une démocratie. Pis, laissées à elles-mêmes, les forces politiques auront très probablement recours à la violence. Si personne ne nie que l'État a trop souvent recours à une violence qui, autour du moment élections, n'est pas légitime, l'on semble ne pas intégrer dans la démonstration le fait que presque tous les acteurs intervenant sur le champ politique adoptent les mêmes pratiques. Sans une ingérence massive (de certaines institutions) d'un appareil d'État autonome par rapport à la société, il n'y aura pas d'élections libres. Une démocratisation supposerait une supervision effective voire pesante par la justice, avec des décisions contraignantes, et avec la capacité d'annuler les élections autant de fois que nécessaire. Et une supervision par la justice suppose un développement des effectifs et une amélioration des performances de la magistrature.

\section{QUE SAIT-ON DES ÉLECTIONS ÉGYPTIENNES?}

1- C'est un moment, qui dure quelques jours ou quelques mois, où l'État n'a plus le monopole de la violence légitime. Je veux dire par là deux choses différentes : d'une part, sa violence quand il intervient dans ce processus n'est pas perçue comme légitime. D'autre part, la violence est, sinon structurelle, du moins une ressource que tout le monde utilise. ${ }^{1}$ La violence est un des, voire,

1. L'équité m'oblige à signaler que le Parti pensant être «le choix naturel » des électeurs a dans l'absolu intérêt à ce que cette violence soit minimale, pour inciter les gens à la participation. Je dois ce point à Marie Vannetzel. Conversation avec l'auteur (mai 2007), et voir Frères éligibles : étude de la mobilisation électorale islamiste en Egypte, le cas des élections législatives de 2005, mémoire de master sous la direction de Gilles Kepel, Institut d'Études Politiques de Paris, 2007. En l'occurrence, il s'agit des Frères musulmans. Mais il reste que le parti doit assurer la protection de ses électeurs et garantir leur accès aux bureaux de vote, et que, pour cela, la violence est nécessaire. 
le langage des élections. Bien sûr, il y a des degrés dans celles-ci. Certaines sont comiques: le retrait systématique, dans certaines circonscriptions, des permis de bus transportant des électeurs d'un candidat de l'opposition. ${ }^{2}$ D'autres, on s'en doute, sont mortelles. Entre ces deux extrêmes, toute une panoplie de procédés existe. Je peux proposer un « catalogue » des violences répertoriées. II sera certainement non exhaustif. Déchirer les affiches d'autrui, attaquer les réunions électorales de l'adversaire, interdire l'accès aux bureaux de vote, frapper les électeurs, etc. Bien sûr, il y a des zones réputées pour leur violence, c'est-à-dire qui sont le théâtre, à chaque élection, de violences. D'autres, plus rares, sont en général calmes. Je signale que quelques observateurs ${ }^{3}$ estiment que les élections comportent deux nouveautés :

- Lors des élections parlementaires de 2000, la violence en Haute-Égypte était plus récurrente et plus intense que dans le Delta. En 2005, cela a été plutôt l'inverse ;

- La violence, lors des élections précédentes, était clanique ou tribale. Depuis 2005, elle est le fait de nervis et de voyous « connus des services de police », payés pour cela, ce qui n'était pas le cas auparavant - je ne suis pas certain que ce soit aussi nouveau. De cette généralisation, de cette privatisation de la violence (pour parler le langage des politistes), il s'ensuit que les "forces gouvernementales", pour prévaloir, doivent mobiliser une masse critique de policiers ou de nervis, ou des deux. Or, même si le ministère de I'Intérieur est le premier employeur du pays, il ne peut le faire partout. Si j'en juge par ces dernières élections, il choisit de porter ses efforts dans les circonscriptions des caciques du parti, surtout s'ils sont bien en cour, ou dans celles des députés ou des personnalités d'opposition qui ont vraiment irrité. Mais déployer de grands efforts n'est pas forcément garant du succès. J'ai longtemps cru que Youssef Wâlî Ministre de l'Agriculture de 1982 à 2004, a été battu en 2005 parce qu'étant en disgrâce, mais les articles des journalistes couvrant sa circonscription relatent des efforts constants des forces de l'ordre pour faire battre son concurrent, Frère musulman.

2- Le chercheur Hasan Abû Tâlib ${ }^{4}$ a tenté de dresser une carte et un calendrier de la violence en 2005. Il s'est fondé sur les articles de presse et sur les rapports des ONG. Je résume ses conclusions : dans cinq circonscriptions, toutes urbaines, la violence précède même l'ouverture de la campagne avec

2. Par exemple à Suez. Voir al Usbû́ du 28/11/05.

3. Cf. Hani Raslan, al-mushâraka al-siyâsiya fil intikhâbât al parlamâniya li 'âm 2005 (La participation politique aux élections parlementaire de 2005) dans : 'Amr Hâshim Rabî' (éd.), Intikhâbât majlis al-sha'b 2005 (Les élections législatives de 2005), Al-Ahrâm, le Caire 2006, p. 199-222, 567 pages.

4. Cf. Hasan Abû Tâlib, Al 'unf fil intikhâbât al parlamâniya (La violence dans les élections parlementaire) dans : 'Amr Hâshim Rabî', op.cit., p. 325-376 et sq. 
la présentation des candidatures. Lors de la campagne officielle, 15 gouvernorats sur 26 et 48 circonscriptions sur 222 ont été le théâtre de violences. Sur ces 48 circonscriptions, seules 9 sont en Haute Égypte. Deux candidats sont tués - le procédé est par deux fois identique : ils sont renversés par une voiture. 67 cas de recours à la violence sont répertoriés. Une petite moitié consiste en des bagarres généralisées avec recours aux nervis. Il convient de noter que Le Caire n'est pas «moins violent» que le reste du pays, puisque 13 cas sur 67 ont lieu dans la capitale (20 si on inclut Guiza). Lors du premier tour du premier round d'élections de 2005, 55 circonscriptions sur 82 ont été le théâtre de violences. 90 cas de violence commis par des nervis contre des groupes d'électeurs ont été recensés. Il y avait, pour la seule circonscription du Bâjûr (Minûfiyya), 25 actes violents, dont des kidnappings de militants et de partisans du Wafd par leurs adversaires. Et il convient de relever que lors de ce round les forces de l'ordre ont peu violé la loi, même si on peut leur reprocher d'avoir laissé faire. Lors du second tour du premier round, la moitié des violences répertoriées (69 sur 138) consiste en des bagarres généralisées avec recours aux nervis, et $13 \%$ de ces violences (16 sur 138) sont des interventions des forces de l'ordre en faveur d'un candidat. Lors du second round des élections, 58 circonscriptions sur 72 sont le théâtre de violences, la palme de la violence revenant à deux circonscriptions d'Alexandrie. Plus généralement la carte de la violence change, le cas de figure le plus fréquent n'est pas la bagarre généralisée avec recours aux nervis (ce cas de figure ne se retrouve que dans 132 cas sur 357), cédant la première place aux actes entravant l'action des commissions supervisant le vote (154/357). 34 blocus de bureaux de vote par la police, interdisant l'accès aux électeurs, sont mentionnés. Et surtout, il y a sept morts. Lors du second tour de ce second round, 47 circonscriptions sur 68 sont le théâtre de violences. 592 cas de violence sont recensés ${ }^{5}$, dont 451 "émanant » de la police et des forces de l'ordre. ${ }^{6}$ Il y a aussi des morts, encore que les versions divergent sur leur nombre, qui oscille entre 1 et 5. Quelques heures avant l'ouverture des bureaux de vote, 665 militants Frères sont arrêtés, notamment ceux qui devaient " observer» le décompte des voix. Lors du premier tour des élections du troisième round, 54 circonscriptions sur 68 sont le théâtre de violences. Il y a deux morts et 514 cas de violence recensés, dont 387 émanant de la police ${ }^{7}$, et 100

5. 148 dans le gouvernorat de la Buhayra, 122 dans celui d'Alexandrie (dont 71 dans une seule circonscription, et 44 dans une autre), etc. Par contre, dans le gouvernorat de Port Saïd, seuls 18 cas de violence ont été recensés.

6. 314 interventions dans le travail des bureaux de vote, 137 blocus interdisant l'accès du bureau de vote aux électeurs. 124 bagarres avec utilisations de nervis.

7. 269 ingérences dans le travail des bureaux de vote, 118 blocus interdisant l'accès du bureau de vote aux électeurs. 
bagarres généralisées avec utilisation de nervis. Lors du second tour, 28 circonscriptions sur 52 sont le théâtre de violences, et il y a six morts. 418 cas de violences sont recensés, dont 388 du fait de la police. ${ }^{8}$ II n'y a que 24 bagarres généralisées avec utilisation de nervis. ${ }^{9}$

3- Dans ces conditions, déplorer le faible taux de participation est une absurdité. Celui-ci, vu le contexte et les risques, est élevé. Et il serait plus élevé si les forces de l'ordre laissaient les électeurs voter.

4- Je voudrais souligner que cet état de fait handicape lourdement d'éventuels libéraux. Je sais confusément, sans être en mesure de le prouver, que les savoirs de la population sur les élections jouent en défaveur du libéralisme. Précisons : on connaît beaucoup de personnes qui prêchaient ou prêchent le libéralisme politique et/ou économique, les droits de l'homme, en ville, et qui sont des féodaux de la plus vile veine à la campagne : I'on peut dénoncer la schizophrénie. Je préfère souligner les nécessités structurelles. On ne gagne pas les élections en étant civilisé.

5- La question de la violence incite à poser la question de la volonté étatique de frauder. On sait que le régime n'obtient plus, depuis longtemps les résultats qu'il souhaite voir apparaître, sauf lors de l'organisation de référendums. Il faut donc poser la question : y a-t-il une "volonté » d'organiser des élections libres? En d'autres termes, les dérapages, qui sont une constante des élections égyptiennes depuis 1923, étaient-ils voulus ou involontaires? Les deux thèses peuvent être plaidées. Bahiyy al Dîn Hasan ${ }^{10}$, dans une journée d'études organisée par le think tank égyptien International Center for Future and Strategic Studies (ICFS) fin 2005, I'a montré avec brio. Selon

8. 209 blocus interdisant I'accès aux électeurs, 179 ingérences dans le travail des bureaux de vote.

9. Et il convient de garder à l'esprit que ces élections sont moins violentes que celles de 1995 qui avaient fait 50 victimes. Celles de 2000 sont relativement plus calmes que celles de 1995 (il y a quand même beaucoup de morts : un pendant la campagne électorale, 3 lors du premier round, 4 lors du second, 7 lors du troisième) mais la tendance est la même. Le propos doit être précisé : en 2000, avant les élections, les forces de l'ordre saisissent, à titre préventif, les armes de plusieurs clans. Quelquefois, ces clans résistent et des échanges nourris de coups de feu ont lieu. En $d^{\prime}$ autres termes, une partie de la violence intervient avant le jour du scrutin. Pour plus de détails, comparer entre I'article d'Abû Tâlib, dont ce paragraphe est un résumé, et celui de Minîsî, "Al 'unf fil intikhâbât » (La violence dans les élections) dans : Hâla Mustafa, Intikhâbât majlis al sha'b 2000, Les élections législatives de 2000, Al-Ahrâm, le Caire 2001, p. 209-226, p. 199-222, p. 211 et sq., 355 pages.

10. Journaliste égyptien et une des figures de proue du champ des ONG et des militants des droits de I'homme. Il avait écrit en 1984 un essai très intéressant sur les élections (Les dimensions du jeu électoral). 
lui, les présomptions sur l'existence au sommet de l'État et du Parti national démocrate (PND) d'une volonté de frauder ou au moins de fausser le jeu sont nombreuses : la composition du comité supérieur supervisant les élections, I'octroi aux candidats PND, et à eux seuls, d'un "symbole électoral » unifié, le refus de la présence d'observateurs internationaux, les harcèlements d'observateurs locaux, la falsification volontaire des listes d'électeurs. Certaines bizarreries, (par exemple la présence sur la liste d'électeurs morts) peuvent s'expliquer par l'incompétence administrative, mais pas toutes (le fait que les partis d'opposition ne reçoivent jamais de bonnes listes, le fait que les listes soient changées - en 2000 et en 2005 - d'un round électoral à l'autre), le phénomène des enregistrements collectifs sur les listes, toujours en faveur des candidats du PND, de fonctionnaires ou de salariés œuvrant pour un candidat PND. Le non-respect des jugements des tribunaux administratifs, le phénomène dit, de manière un peu impropre du "bulletin tournant ${ }^{11}$, qui permet de contrôler les électeurs. Et, bien sûr, I'intervention de la police. L'intervention violente de la police, selon Hasan, n'interviendrait, en 2005, lors des deux premiers rounds (ce n'est plus vrai pour le troisième), que dans les circonscriptions où les Frères ont une chance de gagner. Et enfin la bizarrerie totale des opérations de dépouillement des votes. Hasan pose une autre très bonne question : qui supervise le déroulement des élections? En théorie, il y avait en 2005 un comité supérieur pour ce faire..., mais ce dernier n'avait aucune compétence décisionnelle, il se contentait de donner sur quelques points son avis... Quelles étaient ses compétences et attributions ? Entre autres, définir les règles et critères de la préparation des listes électorales, de leur mise à jour, révision, etc. Mais ce comité ne supervisait pas le processus de préparation/ révision desdites listes et n'avait aucun moyen, aucune ressource pour le faire. Il émettait des propositions relatives au découpage des circonscriptions, mais ces propositions n'étaient pas obligatoires... En ce qui concerne l'organisation des bureaux de vote, la constitution des équipes supervisant le vote, c'était du ressort du président du comité - le ministre de la Justice, en coordination avec le ministère de l'Intérieur... mais aucun rôle n'était reconnu aux autres membres du comité. Sa fonction semblait donc se réduire à la gestion des négociations avec la magistrature et avec les ONG et autres « observateurs » d'élections... En fait, on ne sait pas avec exactitude qui a géré les élections... plusieurs acteurs, dont beaucoup affiliés au ministère de l'Intérieur..., et il était donc difficile d'évaluer la performance des uns et des autres. Mais je pense qu'une étude attentive des articles de journaux permet d'établir avec certitude qu'en 2005 les juges jouaient un rôle crucial, même s'il n'était pas décisif. De leur attitude vis-à-vis des pratiques policières dépendent beaucoup de choses. Par exemple : à Ghurbâl, Alexandrie, lors du second tour, le candidat

11. À ce propos, voir les explications d'Iman Farag dans le présent numéro. 
Frère musulman Usâma Jâdû (qui finissait par gagner les élections) appelait au secours le juge, les flics avaient bouclé les bureaux de vote et interdit l'accès. Le juge Khudayrîn ${ }^{12}$ intervenait et a réussit à "ouvrir » la voie aux électeurs. Par contre, toujours à Alexandrie, au bureau de vote du quartier "King », on avait empêché les femmes de voter, on renvoyait les représentants des partis politiques, et ce avec l'accord du juge. À al-ĉAmiriyya c'étaient les nervis, les baltajîs, qui font le sale boulot, et les juges sont de connivence. ${ }^{13}$

6- Aller voter, c'est partir en guerre. Précision : ce n'est pas forcément aller sur le champ de bataille. L'on peut mener une guerre sans jamais rencontrer la bataille. Les obstacles peuvent n'être que des harcèlements bureaucratiques. Mais l'éventualité de se retrouver au milieu de la violence n'est pas d'une probabilité faible. La presse pro-gouvernmentale avait fait grand cas du fait qu'à Madinat Nasr (Caire), les cadres Frères musulmans aient « fait la prière de la guerre ». Personnellement, je ne suis pas certain d'avoir trouvé cela absurde.

7- Corollaire : aller voter n'est souvent pas une affaire individuelle. L'électeur qui se rase ou qui taille sa barbe, descend de chez lui et va voter, tout seul, comme un grand, existe probablement. II n'est pas majoritaire. Son existence implique la nécessité d'un service d'ordre musclé. Reste qu'en général on va voter en groupe. ${ }^{14}$ Le tout est de savoir si c'est un groupe de militants appartenant à un parti, d'ouvriers travaillant pour tel candidat, de fonctionnaires de telle administration dirigée par tel homme politique, d'habitants du même village

12. Il est à l'époque le Président de l'ordre des juges d'Alexandrie (il perdra les élections en 2007). Il appartient au " courant » de " l'indépendance de la justice », autrement dit aux juges qui militent pour une séparation réelle de l'exécutif et du judiciaire. La presse pro-régime l'accuse d'être un membre de la Confrérie des Frères musulmans. 13. Al-Usbû', 28/11/05.

14. Voir les remarques de 'Aliy al-Dîn Hilâl (un politiste éminent, ancien ministre de la Jeunesse et des sports, figure de proue du PND, il passe pour un allié de Gamal Moubarak et de ses clients) et de Rafîq Habîb (un intellectuel copte protestant, proche de la mouvance islamiste. Depuis quelques années, il passe pour être un des conseiller du Guide suprême de la Confrérie des Frères musulmans, et, auparavant, il a fait partie des fondateurs du " parti » Wasat, islamiste modéré qui n’a jamais été légalisé) lors de la journée d'études organisées par I'ICFS. Hilâl ne développe pas. Rafîq Habîb a émis quelques remarques pertinentes :

- Il faut, pour évaluer les élections, oublier le troisième round, il y a eu trop de fraude. II ne faut pas oublier que la Daqahliyya et la Sharqiyya sont les bastions Frères, et malgré cela ils ne font que trois cent mille voix... ce n'est pas crédible, estime-t-il, à juste titre. Il affirme aussi que les Frères ont eu recours à la " concentration d'électeurs ", à la constitution de packs, mais non à la violence... Je suis sceptique sur ce dernier point, d'autant plus qu'il reconnaît que les Frères ont eu recours à ces mobilisations pour interdire l'accès aux autobus pro-gouvernementaux. 
voulant pour l'honneur de ce dernier que le candidat originaire de ce village remporte l'élection dans la circonscription, que le candidat issu de telle tribu soit le député, etc. Bien sûr, les logiques sont multiples et différentes. II faut, entre autres, distinguer les solidarités primordiales des autres. D'autant plus que I'on ne sait pas si l'érosion des structures patriarcales autoritaires, ou celle des solidarités familiales ou claniques, a des incidences ou non sur le vote, et selon quelles modalités. Je relève, par exemple, que certains auteurs minimisent l'impact de ces érosions, ou plutôt insistent sur la structure paternaliste et autoritaire de la société, affirmant, entre autres, que les personnes susceptibles d’y échapper ou d'entrer en rébellion contre, c'est-à-dire les fonctionnaires ou les jeunes, ne composent qu'une minorité au sein de l'électorat, et qu'ils sont enclins à ne pas aller voter. Cela me semble discutable et devrait de toute façon être testé. ${ }^{15}$

8- Corollaire du corollaire: cela rend la fraude facile. Lisons la presse. Partout le thème est le même. On sait que dans tel bureau de vote tout le monde votera pour tel candidat. S'il est celui du gouvernement, on sera laxiste, on ne vérifiera rien, etc. (sauf si les juges s'acquittent consciencieusement de leur tâche, voir infra). Si, par contre, tel bureau de vote est situé dans une région qui vote «mal », on rend difficile voire impossible l'accès aux bureaux. Le vote collectif permet les opérations de fraude autres que le bourrage des urnes. Bien sûr, dans certains bureaux de vote, la situation est moins claire, en ce que les électeurs peuvent voter pour deux ou trois candidats. Mais comme ils viennent en groupe, ils sont identifiables, d'autant plus que dans les localités les gens se connaissent.

9- La violence obéit à des variables et induit des comportements que l'on ne cerne pas toujours très bien. Je m'explique : dans un collectif publié par I'université du Caire ${ }^{16}$, Mustafa 'llwî rappelait que le déroulement du vote lors

15. Dans le même ordre d'idées, des chercheurs développent des études ou des théories sur la corrélation entre la « culture politique » et le comportement électoral. Selon eux, le « rapport au temps » égyptien serait peu propice à l'éclosion d'une culture démocratique, en ce que les Égyptiens ne voient pas qu'ils peuvent «peser » sur le futur, leur culture étant fondée sur la foi (fataliste) en Dieu et privilégiant le rapport au passé, au moment fondateur de l'islam ou à la gloire pharaonique. Souligner les fragilités et faiblesses de cette argumentation serait facile, mais je préfère faire semblant d'admettre la théorie... pour dire qu'elle n'est plus valable. En admettant qu'elle ait été vraie (chose que je ne fais pas, ne serait-ce que parce que vouloir restaurer le passé est un projet futuriste), ce non-rapport au futur ne pouvait survivre que dans un cadre où le souci du lendemain n'était pas aussi généralisé qu'il ne l'est, ou à un moment où on pouvait raisonnablement penser que les choses iraient en s'améliorant.

16. Mustafa 'Ilwî, (dir.), Intikhâbât majlis al-sha'b 2000, le Caire 2001, 699 p., cf. son article Intikhâbât 2000 muashirât 'âmâ (Élections de 2000 indicateurs généraux) p. 13-14. 
du premier round avait des incidences sur le second : par exemple, le taux de participation en dépend. Certes..., mais il faudrait préciser et éviter les généralisations. On peut, par exemple, imaginer qu'un premier tour s'étant déroulé dans la violence induit une plus faible participation pour le second, mais est-ce certain ? Si, par exemple, la violence a consisté en la complication, voire l'interdiction, de l'accès au bureau de vote, on peut imaginer que des stratagèmes permettant cet accès seront inventés pour le second tour. D'autre part, le même 'llwî rappelait que les élections sans scrutin de liste sont un jeu à somme nulle : un candidat gagne, les autres perdent. II souligne lui-même l'impact de cette règle du jeu sur l'intensification de la bataille électorale.

10- Lié à la violence est le problème de l'arbitraire et de l'obscurité de la législation. Le rapport du régime à la législation peut être résumé ainsi : il n'aime pas reconnaître des droits (et c'est encore plus vrai pour les droits politiques) et n'aime pas se lier les mains. Cela lui permet de frapper s'il le souhaite, même si en général il ferme les yeux, tolère, ou s'accommode de son impuissance. La législation en vigueur combine obscurité, contradictions internes, irréalisme voire absurdité. ${ }^{17}$ Cela entretient l'imprévisibilité et I'incertitude, puisque l'on ne sait pas quand la loi sera appliquée et quand est-ce qu'elle ne le sera pas, et quand est-ce que le régime retiendra telle interprétation et non telle autre.

11- J'ai parlé, supra, de forces gouvernementales. Elles sont plurielles. Je l'ai dit, il est clair que la police ne pouvant être partout (voire pouvant être neutre, j'y reviens), le candidat PND doit compter sur ses propres nervis. Dans le même ordre d'idées, ledit PND s'avère incapable de prévenir le phénomène des candidats "dissidents », c'est-à-dire de personnes n'ayant pas obtenu l'investiture du parti et se présentant néanmoins. Selon un membre du bureau politique de ce parti, les violences fratricides, entre candidats officiels et dissidents, sont souvent les plus dures. Mais moins noté (au moins par moi avant ces élections) est le rôle discret mais souvent décisif des gouverneurs, ces super-préfets aux pouvoirs très étendus. Deux exemples : I'on sait que le gouverneur d'Alexandrie n'approuvait pas le choix des candidats par les instances officielles du Parti au pouvoir. II n'avait pas été écouté. Conséquence : il $n^{\prime}$ « aida pas» lesdits candidats (rendre des services à leurs électeurs ou effectuer des travaux dans leur circonscription) et, selon certains, il favorisa discrètement certains de leurs ennemis. Par contre, celui du Nord Sinaï avait

17. Par exemple, la loi, en 2000, limitait les dépenses d'un candidat à 5000 livres, à l'époque 1500 euros environ, ce qui est clairement absurde. Elle a été remaniée et en 2005 le plafond était de 70000 livres pour le premier tour, ce qui devait être à l'époque l'équivalent de 10000 euros. C'est trop peu dans un cadre structuré par le clientélisme. Le gouvernement peut alors décider de faire respecter la loi ou au contraire de sévir. Les dépenses effectives des candidats dépassent facilement le million de livres. 
été écouté lors du processus d'investiture. Il se battit jusqu'au bout pour les faire élire, exerçant des pressions incroyables (même en tenant compte des normes égyptiennes) sur les chefs de tribus, les notables, etc.

12- Le vote, maintenant : comment l'électeur définit son choix électoral, comment il se comporte. Je sais que les chercheurs privilégient l'étude des réseaux clientélistes, et parlent en termes de clientélisme. Quand en 1991, j'avais effectué mon terrain " discussion avec des électeurs ", j'avais découvert ce "pattern», mais aussi un autre, que j'appelle le vote identitaire. Le vote identitaire, je le décris ainsi : l'électeur perçoit l'élection comme une question qui lui est posée, question qui, dans son esprit, ne s'énonce pas comme étant : "pour quel programme votes-tu, ou que veux-tu? », mais comme étant : " qui es tu, comment témoignes tu ?». (le terme shihâda est de tout temps employé pour désigner l'acte de voter) Et, avant 1952, la réponse à cette question était : "Je suis Égyptien patriote ou nationaliste, donc je vote Wafd». Les adversaires du Wafd pouvaient faire valoir l'incompétence et la corruption scandaleuse, cela ne servait à rien. En 2005, cet automatisme a bénéficié aux Frères musulmans. En d'autres termes (la formule est de ma collègue Selma Belaala), on vote pour un drapeau. J'ai testé cette idée devant un jeune chercheur, Marie Vannetzel, qui a émis quelques réserves pertinentes sur cette analyse. Une autre manière de décrire le phénomène serait la suivante ; depuis 1923, quel que soit le moment, un seul parti détient le langage capable de transcender les classes sociales, les disparités géographiques, attirant massivement les électeurs non inscrits dans des réseaux clientélistes. De 1923 à 1952, ce parti était le Wafd, qui parlait le nationaliste. Depuis 1987, ce sont les Frères musulmans, qui parlent le politico-religieux. Quelle que soit la formulation que l'on retient (je ne suis pas satisfait de mes tentatives pour décrire le plus qu'avait le Wafd et celui dont bénéficient les Frères), il convient de voir qu'il ne faut pas construire une dichotomie. II serait tout à fait faux de penser que le PND a le monopole du vote clientéliste et les Frères celui du vote «trans-classe » ou " identitaire », et il est tout à fait faux de penser que les deux patterns sont exclusifs l'un de l'autre.

13- II convient d'ajouter quelques considérations sur la notion et l'histoire de clientélisme. On l'a déjà dit, les solidarités " primordiales» ne sont pas les réseaux clientélistes professionnels, par exemple. Mais je dois ajouter que les réseaux clientélistes ont évolué, pour s'effriter. D'abord, sur le long terme, le phénomène n'est plus tout à fait le même. Les réformes politiques et sociales de Nasser ont consisté, dans l'ensemble, à accroître les ressources des « clients » contre les patrons, des "dominés » contre les dominants. Un paysan, avant 1952, peut être expulsé à n'importe quel moment s'il n'est pas propriétaire. Cela n'a plus été vrai jusqu'à la fin des années 1990, et cela change la donne. Mais il y a plus important pour notre propos. Tous les développements récents ont pour conséquence un effritement des réseaux clientélistes. D’abord, le fait de privatiser fait perdre à l'État des clients. Mais en plus, du fait de la détérioration des conditions de vie des Égyptiens, il y a le fait massif suivant: 
les demandes des «clients » ont changé, et les ressources des candidats ne peuvent pas toujours suivre. Un député, issu d'une grande famille de Haute Égypte qui a tout au long de l'histoire parlementaire égyptienne (sauf en 2005) des représentants au Parlement me disait au début des années 1990 : "Les notables ruraux sont en train de reculer au bénéfice des hommes d'affaires. Jusqu'à très récemment, les personnes qui venaient demander des services demandaient des crédits pour négocier tel cap. C'était gérable. Mais maintenant, non seulement les demandes de crédit sont de plus en plus nombreuses alors que je ne suis plus aussi riche, mais surtout, plus grave, la demande la plus fréquente est autre: les gens vous demandent de trouver des emplois pour leurs enfants diplômés, et cela je ne sais pas faire ». J'ai soumis, en 2004, le diagnostic à un de ses collègues, un député issu de la police, représentant une circonscription située dans le Delta, qui a confirmé et précisé le propos : «Ouais...il avait raison. Mais c'est plus grave: des emplois, je pourrais en trouver, j'ai un bon carnet d'adresse, question hommes d'affaires. Le problème est qu'ils veulent des emplois dans la fonction publique, et cela c'est devenu impossible ». ${ }^{18}$ Emploi mal payé, mais moins précaire et donnant accès à divers filets de sécurité. Le député poursuivait : "Par contre, je suis content, j'ai réussi à faire faire par l'administration des travaux publics relatifs à l'électricité et à l'eau courante dans ma circonscription ». Ce qui me mène à un autre point, évoqué, également par Sarah Ben Nefissa. ${ }^{19}$ Le savoir conventionnel, il y a une quinzaine d'années, déclinait la maxime suivante : l'aide caritative directe, à telle personne dans le besoin, doit être réservée aux plus démunis, sinon cela passe pour de l'achat de voix. Pour se faire élire, il faut rendre des services " collectifs»: financer la construction d'une école, d'un dispensaire voire d'un hôpital, d'une mosquée. Cela montre un attachement au « quartier » et à ses habitants sans être de la corruption. Or le député dont je parle (le général de police) qui pensait avoir " réussi » ce type de prestations collectives a été battu. Plus généralement, les élections de 2005 ont été le théâtre de l'achat massif de voix. L'achat de voix n'est pas le stade suprême du clientélisme, mais le signe le plus sûr de sa déréliction. La " transaction» se substitue, au moins partiellement, au «lien», aussi intéressé que ce dernier ait pu être. Le phénomène est moins nouveau qu'on ne le dit (il date de la Monarchie, surtout aux moments de crise économique et sociale grave)

18. Selon Al-Dustûr du 07/07/07, les députés demandent une action immédiate du gouvernement pour répondre aux... 75000 demandes de nominations de diplômés qu'ils ont présentées. Le journal parle de centaines de demandes «tombant » sur les ministres et les conseils régionaux. C'est une demande signée par 35 députés, dont la plupart sont du PND ! Ils demandent aussi aux ministres de résilier les contrats de leurs conseillers si bien payés, pour débloquer des crédits et de la place pour les jeunes.

19. Conversation avec l'auteur. 
mais sa fréquence prend des proportions inquiétantes... II convient toutefois de noter que certaines évolutions, décrites par des observateurs perspicaces mais qui doivent encore être confirmées par des recherches ultérieures, vont dans le sens du renforcement des réseaux clientélistes. L'ancien président de I'ordre des journalistes, Makram Muhammad Ahmad, déplorait, lors de la journée d'études de I'ICFS, I'apparition de deux nouveaux types d'électeurs, l'électeur payé (il se trompe, ce n'est pas nouveau), mais aussi des femmes inscrites sur les listes en groupe et qui obéissent au doigt et à l'œil au 'umdah (le maire du village). La forte participation féminine ne serait pas, s'il a raison, le signe d'une émancipation des femmes, mais du renforcement de certains liens clientélistes de dépendance.

14- Une des conséquences de la spécificité des enjeux des élections législatives et de la phénoménologie des relations clientélistes a été soulignée par le politiste Hânî Raslân : ${ }^{20}$ les classes moyennes et supérieures urbaines ont tendance à moins participer aux élections que les autres couches de la société. Pour des raisons simples: d'une part elles ont moins besoin des prestations que peut fournir un député, d'autre part les chances de leurs membres d'avoir (par des voies informelles) accès soit à ce dernier soit à des interlocuteurs au sein de l'appareil d'État sont supérieures à celles des couches défavorisées. Ce phénomène peut être atténué par le « vote idéologique » ou par des spécificités locales, mais il est réel. L'on a ce paradoxe : les couches qui sont le plus attirées par et le mieux informées sur la vie politique sont celles qui ne votent pas, d'autant plus que ce qui les intéresse - politique étrangère et nationale ne se décide pas vraiment au Parlement.

15- En 2005, les Frères ont développé leurs réseaux clientélistes et ont réussi à les mobiliser dans la bataille électorale. Ils ont disposé de plusieurs relais dans la société égyptienne. Par exemple, quelques 18 mois après les législatives, I'hebdomadaire indépendant Sawt al-Umma, dans son édition, du 26/02/07 relate un nouvel épisode de la guerre entre PND et Frères. Le ministre de la Solidarité sociale, 'Alî Musilhî, a promulgué un décret " secret », de dissolution des "buyût al mâl » (Comités des fonds), et des conseils d'administration de gestion des comités de la zakât, à l'échelle de la république. De nouveaux comités de la zakât seront formés, sous la supervision du réseau associatif et du PND. Des mesures sont adoptées par la Banque Nasser pour dissoudre lesdits conseils d'administration. Cela s'inscrit dans le cadre d'un plan dressé par le comité des politiques de Gamal Moubarak, histoire de se donner une crédibilité religieuse et sociale. La décision a été prise, après qu'il s'est avéré que les comités de la zakât ont « orienté » leurs ouailles (32 millions

20. Cf. Hânî’ Raslân, "al-mushâraka al-siyâsiya fil intikhâbât al parlamâniya li 'âm 2005 » (La participation politique aux élections parlementaire de 2005), art.cit, p. 217. 
d'Égyptiens bénéficient de leurs services) vers un vote Frère. Les études de terrain expliquent le vote en faveur des Frères par le volontariat de ces derniers, par leur travail caritatif, et par leur supervision des caisses des buyût al mâl et des comités de zakât. ${ }^{21}$ Il y a 5500 comités de zakât en Égypte, supervisés par 90 agences de la banque Nasser. Voici comment les choses se passent, selon le journal : la banque reçoit des demandes de constitution de ces comités, émanant des villages et des mosquées dépendant de telle agence ou de telle autre. On pressent pour la gestion des personnes réputées pour leur intégrité et leur compétence...ces personnes sont souvent des Frères. Dès que ce comité est agréé par la banque, il reçoit des dafâtir îsâlât (cahiers de reçus) avec le khitm (sceau), que l'on peut donner au pourvoyeur de la zakât. Jusqu'en 2000, ces comités agissaient normalement sous la houlette de la banque Nasser. Puis on a constitué un comité supérieur pour les questions de la zakât à la banque, dirigé par le PDG de la banque, on a défini une stratégie de sensibilisation des riches et de réutilisation des fonds, notamment pour créer des emplois en investissant. Cette nouvelle stratégie a été un succès éclatant, augmentant popularité et ressources des personnes gérant les fonds de la zakât dans les campagnes. 30800 entreprises ont été crées avec cet argent, sans parler des prestations pour pauvres..., dispensaires, écoles, salles de fêtes, orphelinats. La valeur des projets se monte à 32 millions de livres, 33 millions de livres ont été consacrées aux orphelins en 2003, 41 millions de livres données aux familles pauvres à la rentrée scolaire, 120000 valises ont été préparées pour le Ramadan etc. On peut continuer..., mais le résultat est simple : les Frères se sont infiltrés là, notamment dans le Delta, alors que le PND est absent.

16- Dans le même ordre d'idées, la jam'iyya shariyya ${ }^{22}$ a joué un rôle important dans l'ascension des Frères lors des dernières législatives, puisque tous ses comités régionaux ou la plupart d'entre eux sont contrôlés par des Frères. Par exemple, un article, publié par Al-Masrî al-Yawm le 17/11/05, analyse brièvement les élections dans le gouvernorat de Minia. ${ }^{23}$ II est intitulé: "Minia : les résultats prouvent les mauvais choix du PND ». Le journaliste attribue la réussite des Frères à leurs succès dans leurs actions sociales, leurs projets de takâful ijtimâ̂̂̂ (solidarité), aux aides financières, à leurs distribution de repas,

21. Ces buyût et ces comités dépendent de la Banque Nasser, après avoir dépendu du ministère des Finances jusqu'en 1972. Elles « ramassent » trois milliards de livres : les dépôts des Égyptiens se chiffrent à 180 milliards et on prélève la zakât dessus, et il y a un milliard de livres d'actifs (de l'argent laissé par des morts sans héritiers).

22. Trois millions de membres, 360 branches fondamentalistes, mais même Nasser l'a ménagée, quitte à renvoyer son patron en 1968. Infiltrée par la Jamâ'a islâmiyya en 1981, les Frères en prennent le contrôle dans les années 1980.

23. Selon le journaliste, le PND est tellement discrédité dans la région que les « indépendants » ne veulent pas rejoindre ses rangs! 
d'habits, de couverture, pendant les années précédentes, et leur exploitation de la jam'iyya shar'iyya pour appuyer leurs activités. Un article d'Al-Fajr du 25/9/06 mentionne cette association caritative dont le membership se chiffre en millions, et qui « bat le ministère des Waqfs (Affaires religieuses) dans la bataille des mosquées ». Les dix dernières années, les prédicateurs de l'association ont réussi à attirer des dizaines de milliers de fidèles, qui viennent écouter leur cours religieux. De surcroît, la jam'iyya vent des cassettes de leçons et contrôle 5000 mosquées, et 4000 hôpitaux et dispensaires. Là aussi, les tentatives de reprise de contrôle ont échoué. L'imâm de l'association, Muhammad Mukhtâr al-Mahdî, a mené une guerre d'usure contre le ministère des Waqfs qui tentait de prendre le contrôle des mosquées de l'association. Mais le ministère n'avait pas les armes et ressources permettant de gagner : ses ulémas sont mal payés, il y a beaucoup de problèmes à gérer. Al-Mahdî a réussi à convaincre le ministère de renoncer à son plan, et de se contenter d'une supervision administrative et d'une subvention matérielle... Les mosquées de la jam'iyya emploient des salariés et des prédicateurs qui ont le profil requis par le ministère, mais qui ont aussi le look de la jam'iyya (qamîs et barbe). La jam'iyya a aussi 36 instituts de formation de prédicateurs, qui étudient les programmes élaborés par les stars salafistes de I'association (Muhammad Husayn Ya'qûb, Muhammad Hassan, Mahmûd al-Masrî). Leurs sermons sur le califat, sur l'héroïsme, sur l'État islamique intéressent plus que ceux du ministère des Waqfs. ${ }^{24}$

17- À la frontière de ce clientélisme ou de ces réseaux, et du vote que j'appelle, faute de mieux, identitaire, se pose la question du "leader d'opinion », de la personne qui peut peser sur le choix des électeurs de tel ou tel quartier. Mentionnons les notables et les chefs de famille, par exemple, mais il convient de poser la question du rôle des ulémas. Des entretiens conduits par Al Masrî al-Yawm fin décembre 2005 avec des anonymes résidant en zones urbaines permettaient une conclusion préalable intéressante, mais qui s'est avérée fausse pour les circonscriptions rurales. En ville, ou au moins au Caire, le député Frère est perçu par ces anonymes comme une expression du désir d'islamisation de la société, ou d'un ethos musulman, mais il est aussi perçu comme I' « anti uléma » : il est possible de discuter avec lui et ses propos sont falsifiables. C'est un politique, non un religieux, tout en étant, sinon un saint homme, du moins un "honnête ». Or, cette construction n'existe pas, on I'a vu, ailleurs : en province les ulémas ont été un fer de lance de la victoire «Frère » en 2005. Je n'ai pu vérifier si d'autres couches professionnelles les professeurs, par exemple - étaient plus prédisposées que la moyenne à ce statut de «leader d'opinion».

24. Pour plus de détails sur la jam'iyya, voir, par exemple, Mahâ 'Abd al-Rahmân, Civil Society Exposed. The Politics of NGOs in Egypt, Cairo, American University in Cairo Press, 2004, p. 140 et sq. 
18- II convient de relever qu'en 2005 le PND a décidé de tenir à l'écart les réformateurs de l'entourage de Gamal Moubarak et de confier les rênes du ou des processus de sélection des candidats à la "vieille garde », supposée connaître «la province » et être dotée du savoir faire dans l'art du maniement de la carotte et du bâton. Cela aura, ultérieurement, permis à Gamal Moubarak de leur attribuer l'échec relatif du parti - encore une fois, ses candidats officiels auront été battus par les candidats « dissidents », c'est-à-dire par les membres du parti qui se sont présentés sans avoir obtenu l'investiture, et par les Frères. Le directeur du « comité pour les questions politiques » des Frères, al- 'Iryân, minimisait la performance de la Confrérie en affirmant que la première force politique du pays était... les dissidents du PND. II convient de ne pas minimiser ce phénomène : une bonne moitié des députés PND ont été élus malgré leur parti et cela change la donne : s'ils ont intérêt à être en bons termes avec le gouvernement, en revanche ils ne doivent rien aux caciques du parti. Évidemment, les choses peuvent être plus compliquées que ne l'implique ma dichotomie : dans ma circonscription (Qasr al-Nîl), I'on avait quelques raisons de penser que le " dissident » du PND, Hishâm Mustafa Khalîl, avait I'appui discret de quelques caciques du parti et de quelques acteurs dits gouvernementaux, soucieux de battre le notable qui avait reçu l'investiture.

19- Ce qui nous mène à la campagne électorale, qu'il s'agisse des discours ou des « incitations » à voter. La presse n'est pas forcément bon guide, elle a tendance à privilégier les bizarreries. C'est ainsi qu' Al- âlam al-Yawm du 28/11/05 publie un article sur la «publicité » pour les candidats, hors « les achats de voix ». D'une part, les candidats ont eu recours au net pour expliquer leur programme, par la création de sites web. Mais au sein de ce répertoire, on repère également l'utilisation des cadeaux symboliques, mais aussi, dans certains cas, de viagras. L'utilisation des téléphones portables et des messages SMS, avec des versets coraniques, la location de sièges de café, I'utilisation d'écrans géants, la publicités dans les journaux, I'organisation de repas collectifs le jour des élections, le paiement de la scolarité des plus démunis voire d'écoles entières, la prise en charge de certains frais (80\% dans un cas) de mariage des jeunes femmes dans certaines circonscriptions, le recours à des sociétés de relations publiques pour gérer la campagne (cela coûte de 50 à 150000 LE). Un candidat (Tal'at al-Sâdât) s'est promené avec un lion dans sa circonscription. En Haute Égypte celui qui a pour symbole électoral le chameau fait sa campagne sur un chameau. S'il perd il fusille ledit chameau, s'il gagne il l'égorge et le mange avec ses partisans ('Ali Hasan, PND). Un candidat s'engage à s'occuper de sa circonscription même après sa mort (waqf pour financer les activités caritatives). Un autre candidat saïdî (de Haute Égypte) a commencé sa campagne par une tournée au Koweït : beaucoup de membres de sa circonscription y travaillent et il leur demandait de convaincre leurs familles de voter pour lui. Après sa victoire, un candidat prend un grand portrait de son adversaire, le met sur un âne et se promène avec lui dans la circonscription. On note également I'utilisation extensive de la musique, de micros, DJ's et d'invitation d'artistes. 
20- Mais les candidats peuvent déployer un certain esprit de méthode. Marie Vannetzel, qui travaille sur les mobilisations de Frères, m'a expliqué la stratégie Frère pour tenter de conquérir Madinat Nasr (Le Caire), circonscription où membres des classes moyennes et privilégiées côtoient les plus défavorisés. Il fallait donc privilégier, en s'adressant aux premières, le discours sur les réformes politiques et sur la démocratisation. Pour les pauvres, discours religieux (variés, mais en général salafistes) et prestations/aides étaient dominants. Elle a également recueilli des informations faisant état de sondages effectués par les Frères pour identifier attentes et craintes. Il y a lieu de penser que cette stratégie a été suivie partout.

21- a) Deux «lieux communs » de la science politique travaillant sur les élections égyptiennes $\mathrm{m}^{\prime}$ intéressent maintenant : le rappel, que tout le monde accepte mais ignore, de la pluralité de ce pays. L'Égypte est un État-Nation que l'on estime homogène, et ce n'est pas faux, mais il l'est beaucoup moins qu'on ne le dit. Rien de commun entre par exemple la circonscription de Shubra al-Khayma, qui regroupe à la fois des zones industrielles et rurales, des électeurs issus de toutes les classes de la société, y compris de riches commerçants, et, par exemple, celle du village de Hawâtka, conglomération de villages du gouvernorat d'Assiout. Dans certains gouvernorats, comme ceux de Qina, de la Mer Rouge et ceux du Sinaï, ou encore celui de Suez, le facteur tribal est important, dans d'autres, il ne l'est pas du tout. Et cette importance se fait sentir selon des modalités différentes : à Qinâ et en mer Rouge l'implantation Frère est faible, mais à Suez c'est le contraire. Il est difficile de savoir si, à Qina, la faiblesse de la présence Frère s'explique directement par les liens de solidarité tribale, ou par le fait que d'importants efforts dans le domaine de l'infrastructure ont été consentis dès le début des années 1960, le gouvernement central s'efforçant d'amadouer les tribus. Certaines villes sont relativement prospères (Damiette), d'autres sont dévastées par les politiques de privatisation (Mihalla). Plus généralement, pour les villes, I'on sait que dans certains quartiers la population est plus ou moins homogène, alors que dans d'autres un distinguo entre familles awlâd al-balad, d'enfants du pays, installées de très longue date et arrivés plus récents est construit par les membres des premières. Lors de pointages effectués auprès de notables alexandrins après les heurts confessionnels ayant accompagné l'enterrement de coptes tués par un déséquilibré dans une église, l'explication qui avait été avancée était que ce « comportement n'était pas alexandrin » et qu'il était le fait d'individus, chrétiens ou musulmans, récemment (quinze ans) rentrés d'Irak! Que l'explication soit ou non bonne elle montre l'existence d'un construit ayant des incidences. Mais seuls des travaux de terrain poussés pourraient déterminer son éventuelle influence sur les processus électoraux. Plus généralement, le quartier d'aujourd'hui n'est plus le même que celui décrit par, mettons Najîb Mahfûz, il y a cinquante ans. Or je crois que les modes de vivre ensemble ont des implications sur les votes clientélistes, et aussi sur les chances de succès des idéologies concurrentes. Sans parler de déterminisme, il y a une forte 
connexion, je pense, entre l'idéologie nationaliste égyptienne et les anciens « modes de vivre ensemble »..., alors que les plus récents favorisent plutôt les islamistes.

22- b) L'autre lieu commun est que les enjeux " nationaux », en tant qu'opposés aux enjeux locaux, n'ont aucune incidence sur les élections législatives. Les programmes des partis n'intéressent pas, la politique économique, encore moins, etc. Cela n'est pas faux mais demande à être précisé. Comme partout ailleurs, en temps normal, et l'Égypte est, même si elle le déplore, dans un temps normal, les considérations « intérieures » priment, la politique étrangère compte peu, et cela est accentué par la présidentialisation autoritaire du pays. Ce que le député pense de la politique étrangère est perçu comme étant sans incidences, puisque sur ce point le président décide seul. Cet état de fait joue contre le parti au pouvoir, puisque la politique étrangère est un des seuls domaines où la population s'accommode, voire est satisfaite, de son bilan, alors que les options de l'opposition peuvent inquiéter. Par ailleurs, Sarah Ben Nefissa avait déjà souligné la dimension "vote sanction » contre le PND lors des élections de 2000. ${ }^{25}$ À juste titre. Mais qui dit « vote sanction » dit forcément, même si ce n'est que partiellement, verdict sur des enjeux nationaux. Plus précisément, la politique de privatisation du secteur public, politique «nationale » qui a de très lourdes retombées locales, est un arrièreplan oppressant dans plusieurs circonscriptions. Plusieurs circonscriptions ouvrières, que I'on dit attachées au nassérisme, punissent les candidats du PND à cause de cette politique nationale..., en votant pour les Frères, ennemis jurés de Nasser. Ailleurs, telle femme candidate proche du PND battra un député Frère, parce qu'entre autres elle a mené un combat sans répit contre la vente de l'usine qui est le principal employeur de la région.

23- Noter I'article de Wahîd 'Abd al-Majîd dans Rûz al-Yûsif le 2/12/05. II relate la victoire de Jamâlât Râfi' contre le candidat Frère à Tûkh, Qalyûb. Le candidat Frère découvre avec horreur, quelques jours avant le scrutin, qu'une femme risque de le battre. Et là tout le vieux discours Frère sur les femmes ressort. "lâ yuflihu qawm idhâ amarathum imra'a ", un peuple est condamné à l'échec s'il est commandé par une femme (selon Al- âlam al-Yawm du 28/11/05, c'est pour séduire les jeunes qu'il dit cela - le même journal indique que Jamâlât (qui, soit dit en passant, porte le hijâb) a distribué un tract, rappelant que Makârim al-Dîrî était une candidate frère, et rappelant la fatwa de 'Alî Jum'a sur la participation politique des femmes, et concluant avec le verset coranique sur ceux qui disent une chose et en font une autre. Jamâlât distribue un tract expliquant hukm al islâm sur les gens qui disent ce qu'ils ne font pas. Elle a fait 27400 voix contre 19000 aux Frères. Le numéro d'Al-Fajr du 5/12/05

25. Sarah Ben Néfissa, Alâ' Al-Dîn Arafat, Vote et démocratie dans l'Egypte contemporaine, Paris, Karthala, 2005. 
publie aussi un long article sur ladite Jamâlât. D'abord ce n'est pas une inconnue, même si c'est la première fois qu'elle devient député. Elle a joué un rôle important pour prévenir la « privatisation » de l'usine Qahâ (un des piliers de I'agroalimentaire nassérien). Elle avait refusé de se laisser corrompre par certains députés. De même, elle s'oppose fortement aux Frères, même si elle est voilée ; la division de la société en « frères musulmans » et « frères kafara (infidèles) » n'est pas pour elle. Pour devenir député, elle a écrasé le candidat Frère mais aussi un célèbre député PND, Mahmûd Ma'rûf. Son parcours illustre beaucoup de bizarreries: d'une part, elle est proche du PND, mais ce dernier ne l'a pas « pressentie » comme candidate, d'autre part, elle est membre du «Conseil national pour la femme » (organisme gouvernemental dirigé par des personnalités prestigieuses et s'occupant des causes féminines) mais ce dernier ne l'a pas appuyée. Or elle avait déjà fait un score honorable lors des précédentes législatives. Et c'était avant l'affaire Qahâ. Noter que dans les indications que l'on donne sur l'usine Qahâ on apprend beaucoup de choses sur l'histoire de la circonscription, comment la construction de l'usine Qahâ a été vécue comme un formidable espoir pour les habitants, qui leur permettrait d'améliorer leur sort. Alors que Jamâlât poursuit ses études secondaires, son père meurt. Elle doit donc gagner sa vie et travaille à Qahâ, tout en préparant un diplôme de tarbiyya istimâ'iyya (éducation sociale). Elle devient assistante sociale. Elle est vite populaire, et d'ailleurs elle monte dans la hiérarchie syndicale. Elle s'oppose à la privatisation de Qahâ, guerroie I'acheteur, qui veut surtout vendre les machines et licencier. Et dans ces élections, les ouvriers la font élire. Son discours sur les Frères est très dur : selon elle, leur pratiques ne sont pas à la hauteur de l'islam. Le candidat Frère est un " architecte » qui a démissionné du syndicat des ingénieurs pour se faire passer pour ouvrier. II a acheté des voix. Il a utilisé le hadîth "la'an Allâh qawman wallû imra'a » (Dieu maudit un peuple mené par une femme). Elle a rétorqué en soulignant ses pratiques, en expliquant le distinguo entre wikâla (mandat) et wilâya (gouvernement), etc. Sur les comptes de sa campagne : elle a coûté 200000 LE, mais elle n'a payé de sa poche que 20 000, les habitants de la circonscription l'ont beaucoup aidée. La liste de ses «thèmes » illustre la dévastation de la campagne : elle se bat pour des égouts, pour l'eau, pour les conflits sur les impôts fonciers. Et, avant tout, les droits des ouvriers. Il convient toutefois de ne pas présenter un tableau trop idyllique de l'exploit. Al-Usbû́ rapporte qu'à Tûkh on a interdit l'accès ou fermé certains bureaux de vote, dans des zones situées acquises au candidat Frère, et que cela a profité à Jamâlât Râfi'. La police menace les "grands des familles » (les grandes familles, je suppose) de la région...qui veulent voter Frères !

24- Je me suis étendu sur cet exemple pour illustrer plusieurs points. II s'agissait d'une part de rappeler que les grands choix de politique nationale ont des incidences sur la vie locale et que les électeurs se prononcent làdessus. D'autre part, cet exemple montre que ce passage du national au local s'effectue selon des modalités qui ne peuvent être prédites : dans ce cas, certes 
tout à fait exceptionnel, le PND s'appuie sur les ouvriers, alors que les traditionnels alliés du pouvoir central, les grandes familles, veulent voter pour les Frères... Dans cette circonscription, le candidat PND était celui des ouvriers. Toujours sur ce passage du national au local, il y a bel et bien un vote copte, mais il ne se déploie pas toujours de la même manière: dans une circonscription où il y avait un candidat copte, les électeurs coptes ont, contre toute attente, voté pour le député sortant musulman : "II a toujours été parfait avec nous, c'est stupide de le sanctionner simplement parce qu'il y a un copte», m'a-t-on expliqué. ${ }^{26}$

25- Il faut poursuivre : selon le savoir conventionnel des acteurs, du moins celui des acteurs que j'ai fréquentés, le «programme de gouvernement » des partis politiques est secondaire. Pis, les inconvénients de la publication dudit programme, selon ce savoir, peuvent être supérieurs aux avantages qu'elle procure. Un programme, ce sont soit des propositions précises, soit des assertions vagues n'engageant à rien. Des assertions vagues ne "font pas sérieux ", des propositions précises peuvent être critiquées et attaquées. Elles doivent forcément mécontenter des secteurs. Elles « engagent » les candidats. Un programme, ce n'est pas un facteur d'union, mais de dissensus. Pourquoi, si tel est le cas, les partis persistent-ils à promulguer un programme ? Même les Frères musulmans, qui, en général, évitaient de sacrifier à ce rituel, ont rédigé le leur. La réponse doit être complexe, et spéculative : je suis enclin à penser que I'on ne peut pas à la fois soutenir que les programmes n'ont pas d'importance et affirmer que les « réfutations des programmes d'autrui » ont, elles, des incidences. Il faut choisir : soit ce champ discursif spécifique est important, soit il ne l'est pas. Je suis donc enclin à donner raison aux acteurs sur le premier point (l'importance des programmes est secondaire), mais pas sur le second (les programmes n'ont que des inconvénients, dont celui de prêter le flanc à la critique). La publication des programmes a quelques fonctions, que les acteurs sous-estiment. D'une part le programme est « itération » de l'identité du parti : il prouve son ancrage, son ethos. D'autre part, il est gage de « professionnalisme », et cela compte. Mes observations de terrain vont dans ce sens : face à certains secteurs de I' " opinion » (urbaine, qui voit les candidats dans des cafés, ou encore certaines couches de ce que l'on appelait il y a quarante-cinq

26. Une des études de cas menées à bien dans le collectif dirigé par Hâshim Rabî' mentionne un cas de figure similaire. Un candidat copte dans la circonscription d'Isnâ (gouvernorat de Qinâ, Haute Égypte) ne réussit même pas à faire le plein de voix coptes. L'auteur de la monographie attribue cela à la non-existence, dans la région, du parti que représentait ce candidat (le Rassemblement progressiste). Cela me semble un peu « court ». Cf. I'article de Sâmih Râshid «Intikhâbât majlis al sha'b 2005 - hâla tatbikiyya (Les élections de l'assemblée du peuple de 2005 - étude d'un cas)». in Rabî' (ed.), op.cit., p. 377-470, p. 387. 
ans les nouvelles classes moyennes), il ne faut pas passer pour un hurluberlu. II y a des " rituels » qui signifient le savoir-faire de l'élu. Ce savoir faire doit être un dosage de "maîtrise du langage clientélaire » et de "capacité à indiquer un cap ». Enfin, à un niveau méta-théorique non falsifiable, je crois que la rédaction et la publication des programmes est une pratique induite par l'institutionnalisation du multipartisme, et qu'elle est devenue une nécessité, même s'il y a des résistances " culturelles », due à une vague méfiance " atavique » à l'égard de ce qui induit, met en scène et incarne la division, le dissensus.

26- Le programme "Frère » pour les législatives mérite un exposé détaillé, que je ne peux conduire dans ce cadre. Je me contente donc de résumer et de compléter le diagnostic du politologue Ahmad Minîssî. ${ }^{27}$ Après avoir relevé que les Frères, contrairement aux autres forces, ont réellement tenté d'assurer à leur programme la diffusion la plus large (distribué par des militants, envoyé à des adresses e-mail, etc.), il constate que les Frères sont les seuls à n'avoir pas distingué " politique étrangère » et " politique intérieure », préférant une classification : "renaissance-développement-réformes ». II critique aussi le vague des propositions Frères, qui, en outre, se prononcent pour une république parlementaire constitutionnelle démocratique, mais qui ajoutent «dans un cadre islamique » : soit I'ajout est, comme le pense Minîssî, dépourvu de sens (l'islam n'a pas de préférences spécifiques pour un type ou un autre d'arrangement institutionnel, se contentant de prôner la justice), soit il désigne le désir d'instaurer une théocratie. II revient plus loin à la charge, pour affirmer que la répartition des responsabilités au sein de l'exécutif, telle qu'elle est effectuée par le programme Frère, n'est pas caractéristique d'une république parlementaire, mais d'un régime présidentiel similaire à la Ve république en France. Le volet économique du programme est intéressant. Mais alors qu'il accumule propositions détaillées sur toutes sortes de questions, la définition des principes d'ensemble du système, des grandes lignes d'action (politique d'expansion monétaire ou de rigueur, par exemple, privatisation ou non) et des objectifs macro-économiques brille par son absence.

Le programme PND mérite une remarque rapide. Après les législatives, le cacique Safwat al-Sharîf, dans un entretien téléphonique avec un journaliste, Sulaymân Jûda ${ }^{28}$, affirme être inquiet de la montée en puissance des Frères. "Pour contrer le discours émotif des Frères, il faut un discours pragmatique, traitant des problèmes des gens, et faisant du terrain (yanzil ila al shâri). Si les Frères montent les gens en interpellant leur foi religieuse, le PND (...) montera les gens en interpellant leur foi patriotique et non leur foi religieuse.

27. Ahmed Minîsî, Al barâmij al intikâbiyya lil ahzâb wa al quwa al siyâsiyya al raîsiyya, (Les programmes électraux des partis et des forces politiques principaux) dans : Rabi' (éditeur) : Intikhâbât majlis al- sha'b 2005, op.cit., p. 231.

28. Qui le rapporte dans Al-Masrî al-Yawm, 06/12/2005. 
La première est toujours plus forte, plus durable, plus stable chez les gens ». D'abord, cette foi en la primauté automatique des passions nationalistes sur celles, religieuses, est caractéristique de générations dont les derniers représentants sont en train de disparaître. Ensuite, le diagnostic est intéressant, mais la prescription illustre l'impasse discursive du PND. Une posture nationaliste serait, dans l'état actuel des choses, une " prescription » normative définissant des postures que le régime ne peut adopter, ou un «agenda » qui ne peut être le sien, ou un refus d'alliances qu'il ne peut formuler. En d'autres termes, et à supposer qu'on puisse par décret substituer un langage et une grammaire nationaliste au langage et à la grammaire religieux en cours, alors que l'on ne sait même pas faire respecter les feux rouges, on n'obtiendrait qu'un nouvel agencement de normes impossible à respecter, agencement qui lui aussi délégitimerait le régime. Le programme PND n'a pas retenu cette solution : plutôt que de s'aventurer sur le terrain des passions identitaires, ou plus exactement plutôt que de $s^{\prime} y$ attarder, il a tenté de se prévaloir de ses réalisations, bref d'instaurer une logique d'évaluation. ${ }^{29}$ Mais les réformes, réelles, se sont traduites par un mal-vivre accru pour pratiquement toutes les couches de la population, sans même réussir à donner l'impression qu'il n'y avait là qu'un "mauvais moment » à passer. Cette solution, notons le bien, n'est pas celle que recommande al-Sharîf. Il réitère la formulation de l'impossible objectif nassérien : I'abolition fantasmée de la distance et des obstacles entre gouvernants et gouvernés, la communication symbiotique avec ces derniers, l'écoute des pauvres, I'informalité de l'oral, de la présence immédiate, du vivre ensemble.... Idéal que partagent tous les acteurs du système, mais qui se heurte aux considérations matérielles : les demandes sont impossibles à satisfaire et la méchante population ne se berce pas de mots.

29. Je dois cette grille d'analyse, " évaluation de bilans versus posture identitaire » à Jean-Noël Ferrié. 